\title{
Direct Contacting with Liquid Electrolyte Facilitates the Surface Phase Transition in Ni-rich Layered Cathode
}

Lianfeng Zou ${ }^{1}$, Wengao Zhao ${ }^{1}$, Haiping Jia ${ }^{1}$, Jianming Zheng ${ }^{1}$, Linze Li $^{1}$, Daniel Abraham ${ }^{2}$, Guoying Chen $^{3}$, Jason Croy ${ }^{2}$, Ji-Guang Zhang ${ }^{1}$ and Chongmin Wang ${ }^{1}$

${ }^{1}$ Pacific Northwest National Laboratory, Richland, Washington, United States, ${ }^{2}$ ANL, Lemont, Illinois, United States, ${ }^{3}$ LBNL, Berkeley, California, United States

The layered-to-disordered rock salt phase transitions in the Ni-rich cathode of Li ion batteries are well recognized to impede the $\mathrm{Li}$ ions diffusion, facilitate the transition metals dissolution, and alter the electron-insulating properties of SEI layers, which contributes significantly to the capacity decay ${ }^{1-5}$. Often, the phase transitions are more frequently observed on open surfaces than in the bulk. Based on the common perception that open surfaces are the first and primary sites undergoing the structure reconstruction, the phase transitions on such sites are routinely selected to demonstrate the severity of cathode structure degradation and a general picture of "open surface reconstruction" is established when referred to the structural degradation in the layered structure ${ }^{6-9}$. However, the fundamental mechanism that governs the interfacial degradation have not been revealed. In addition, open surfaces merely represent the category of solid-liquid terminations, a large population of other characteristic interfaces, for example, solid-solid and solid-gas interfaces, are yet ubiquitous inside cathode materials. Once take place, the phase transitions at GBs can easily modify the electrochemical cycling performance, nevertheless, the critical entities have rarely brought into attention. Herein, high-angle annular dark-field scanning transmission electron microscopy (HAADF-STEM) and Annular bright-field (ABF-STEM) imaging is used to establish phase transitions that occur across various boundaries within cathode-oxide particles having a Ni-rich composition.

The Ni-rich electrode was harvested from the cycled coin cells and the cross-sectional samples were prepared by FIB lift-out on the FEI Helios DualBeam microscope using ion beam (voltage range from 2$30 \mathrm{kV}$ ). STEM observations were performed on $200 \mathrm{kV}$ JEOL JEM-ARM200CF microscope equipped with a probe spherical aberration corrector. The high-angle annular dark-field detector (HAADF) signals were collected from 68 to $280 \mathrm{mrad}$. Various facets of the primary particles were selected for the HAADFSTEM imaging to ensure the observation of intrinsic features.

The STEM results demonstrate that, comparable to the open surfaces that suffers from severe phase transition, the sealed surfaces and incoherent boundaries that are free from the electrolyte attacking undergo much mitigated structural degradation (Figs.1a-b), thereby rationalizing the vital roles of solidliquid interface reactions in controlling the phase transition. On the other hand, the oxygen vacancies governed the phase transition behavior at solid-solid interface: the significant oxygen loss leads to the severe phase transitions at coherent twin boundaries (Figs.1e-f), by contrast, the retain of oxygen results in sluggish phase transitions at incoherent boundaries (Figs.1c-d). The studies establish the correlation between the phase transition and both the intrinsic and external parameters and may provide a general insight into phase engineering via tuning oxygen properties. 

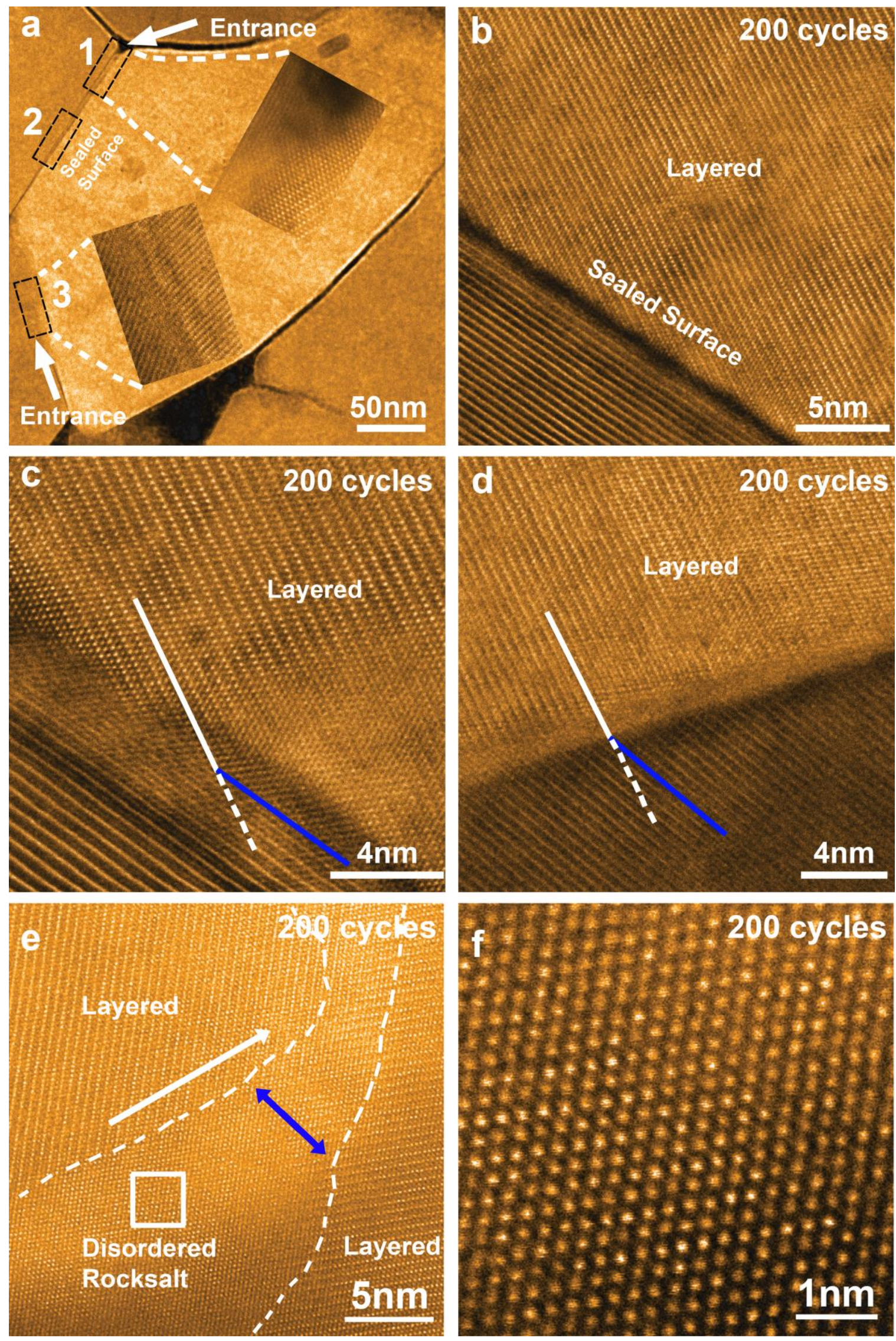
Figure 1. Phase transitions inside the secondary particles. All the HAADF-STEM imaging are performed on the 200 cycled NMC76. (a) The construction of a typical sealed surfaces. The black rectangle 2 outlines the sealed surface area. The black rectangle 1 and rectangle 3 indicate the entrance to the area 2 . The inserts are the zoom in view of the two entrances. (b) Atomic resolution imaging of the sealed surface from rectangle 2 area in a. (c-d) The configurations of incoherent boundaries after prolonged cycling. The white and blue lines indicate the (003) planes of upper and low grains. (e) HAADF-STEM image of twin boundary. The white dashed lines outline the boundary between the layered structure and the disordered rocksalt structure. The white and blue arrows point to the along the boundary, and across the boundary propagation direction. (f) Zoom in view of the twin boundary from the rectangle area in e.

\section{References}

1. Betz, J.; Brinkmann, J. P.; Nölle, R.; Lürenbaum, C.; Kolek, M.; Stan, M. C.; Winter, M.; Placke, T. Cross Talk between Transition Metal Cathode and Li Metal Anode: Unraveling Its Influence on the Deposition/Dissolution Behavior and Morphology of Lithium. Adv. Energy Mater. 2019, 9, 1900574.

2. Gilbert, J. A.; Shkrob, I. A.; Abraham, D. P. Transition metal dissolution, ion migration, electrocatalytic reduction and capacity loss in lithium-ion full cells. J. Electrochem. Soc. 2017, 164, A389-A399.

3. Li, W.; Kim, U.-H.; Dolocan, A.; Sun, Y.-K.; Manthiram, A. Formation and inhibition of metallic lithium microstructures in lithium batteries driven by chemical crossover. ACS nano 2017, 11, 5853-5863. 4. Zheng, H.; Sun, Q.; Liu, G.; Song, X.; Battaglia, V. S. Correlation between dissolution behavior and electrochemical cycling performance for $\mathrm{LiNi}_{1 / 3} \mathrm{Co}_{1 / 3} \mathrm{Mn}_{1 / 3} \mathrm{O}_{2}$-based cells. J. Power Sources 2012, 207 , 134-140.

5. Zou, L.; Li, J.; Liu, Z.; Wang, G.; Manthiram, A.; Wang, C. Lattice doping regulated interfacial reactions in cathode for enhanced cycling stability. Nat. Commun. 2019, 10, 3447.

6. Lin, F.; Markus, I. M.; Nordlund, D.; Weng, T.-C.; Asta, M. D.; Xin, H. L.; Doeff, M. M. Surface reconstruction and chemical evolution of stoichiometric layered cathode materials for lithium-ion batteries. Nat. Commun. 2014, 5, 3529.

7. Maleki Kheimeh Sari, H.; Li, X. Controllable Cathode-Electrolyte Interface of Li [Ni0. 8 Co0. 1Mn0. 1] $\mathrm{O}_{2}$ for Lithium Ion Batteries: A Review. Adv. Energy Mater. 2019, 9, 1901597.

8. Li, T.; Yuan, X.-Z.; Zhang, L.; Song, D.; Shi, K.; Bock, C. Degradation mechanisms and mitigation strategies of nickel-rich NMC-based lithium-ion batteries. Electrochem. Energy Rev. 2019, 1-38.

9. Lin, F.; Nordlund, D.; Li, Y.; Quan, M. K.; Cheng, L.; Weng, T.-C.; Liu, Y.; Xin, H. L.; Doeff, M. M. Metal segregation in hierarchically structured cathode materials for high-energy lithium batteries. Nat. Energy 2016, 1, 15004. 